\title{
Industry 4.0: Key Questions in Manufacturing
}

\author{
Camelia Gabriela Arsene ${ }^{1, *}$, and George Constantin ${ }^{1}$ \\ ${ }^{1}$ University Politehnica of Bucharest, Machines and Manufacturing Systems Department, Splaiul \\ Independenței 313, 060042, Bucharest, Romania
}

\begin{abstract}
The actual industrial revolution is bringing multiple opportunities of increased productivity through Cyber-Physical Systems, better cost control and decision making through Digitization and improved data communication chain through IoT. However, finding the best fit within Industry 4.0 context and writing the business case to support massive investments is not an easy task considering the organisations and the environment dynamics. The risk of disruptive implementation may be also high and involving massive implications. The paper proposes to highlight in a structured way - top-down approach - the key questions to address as an organization when leaving the awareness phase and preparing the implementation. This is a cross-industry study, covering as well different patterns: make to stock, make to order, engineering to order.
\end{abstract}

\section{Setting the scene}

It is already broadly acknowledged that Industry 4.0 represents an actual and large concept unifying breakthrough technologies and equally a tremendous human opening trend in exploring the new manufacturing opportunities.

The Industry 4.0 scene (Fig. 1) is revealing Cyber-Physical Systems (CPS) advanced and accessible technologies with straightforward applicability, Digital Transformation leveraging digital technologies, Big Data collecting, analysing and using data from multiple sources [1], Internet of Things (IoT) for integrated and fast communication within/ between complex systems, Artificial Intelligence and Cognitive Technologies for evaluating in a quick and structured manner and extracting the relevant data for fast diagnoses, improvement detection and decision making. Final target is the increased productivity in an overall economical context with exhausted opportunities.

The overall Industry 4.0 implementation speed is encouraged and fuelled by a powerful partnership having few main actors: Software Companies with fast paced development environment, companies acting in various industries looking for advanced solution to optimize both benefit and operating models and finally, the final customer's expectations to get best compromise between cost, safety, quality, speed and user-friendly experience when getting various products and services.

Some of the new technologies are not totally new, their fundamental concepts being developed in the past. Their recent fast development and introduction was boosted by "computing power" and increased accessibility from cost perspective as mentioned by

\footnotetext{
* Corresponding author: Camelia.Gabriela.Arsene@gmail.com
} 
Delloite in one recent study [2], referring as well to Moore's law. Another trend highlighted by consultants is early adoption advantage, which may only work with a strong and relevant business case model.
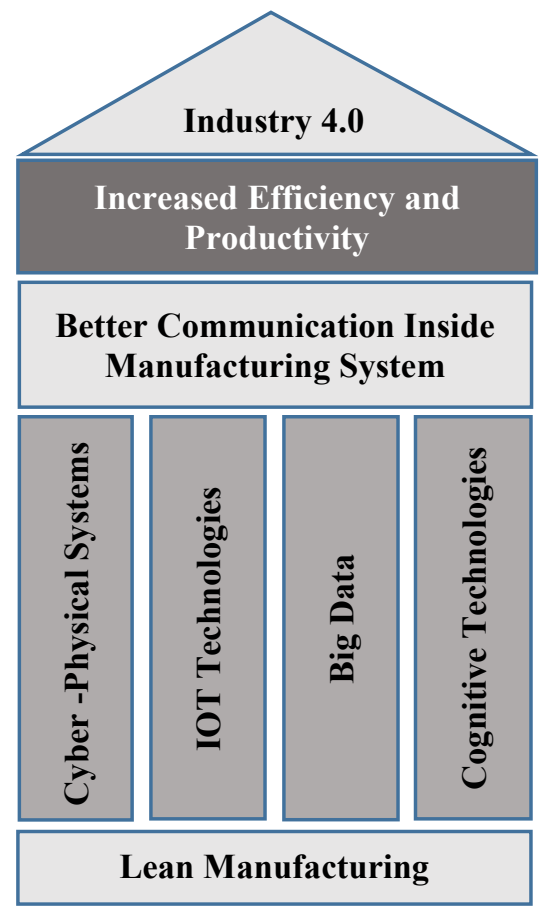

Fig. 1. The Industry 4.0 scene

This fast-paced trend is raising a few key questions that each organization should address before stepping into the implementation phase and the purpose of this article is to address them. The authors propose a top-down approach where analysing first time the best fit, starting from manufacturing environment (as best fit is a strategical choice), then going through some key elements to be considered when business case for best fit is built and finishing with a list with proposed new indicators to be monitored after the implementation. (Fig. 2)
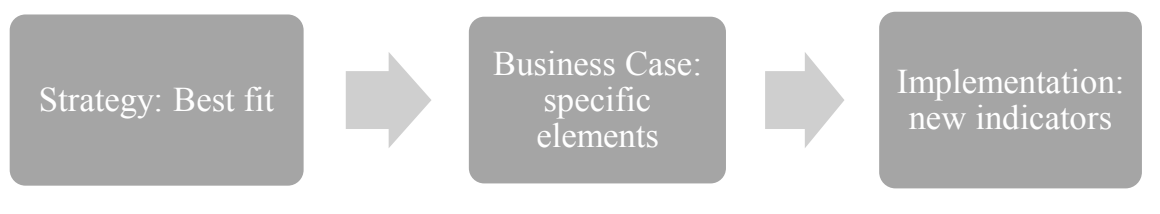

Fig. 2. Industry 4.0 Top Down approach

1. What is the best fit in overall Industry 4.0 context: what Industry 4.0 elements are worth being implemented and when?

Most of the companies are looking after increased benefits (productivity, quality, growth, etc.) and the early adoption of Industry 4.0. is proportional with the magnitude of the results.

However, the choice about what Industry 4.0. element to be implemented and when, must be carefully documented based on various specific industry, market, business, organization and manufacturing environment features. 
The manufacturing environment generally refers to the overall context in which the manufacturing strategy is designed and executed [3]. Considering various manufacturing environment types: from "Make to stock" to "Engineering to order", the decision about Industry 4.0 customization varies.

2. How the business case must capture the new elements to document in the best manner the course of actions, benefit and cost?

The Business Case for various Industry 4.0 elements is not an easy quick task, both benefit and cost being difficult to estimate for a simple natural reason: we are in front of something new. In some cases, the companies prefer to develop the trust by running a pilot and then building larger capabilities and scaling -instead of preparing the classic business case for the large implementation.

However, irrespective of which method is used to get the buy in for implementation course of actions, all implications must be captured.

3. What are the key indicators to be monitored after implementation?

Industry 4.0 brings few major game changers: data, cloud storage and computing, interconnectivity, cyber-physics, AI, etc. Hard to believe that after the implementation and during the surveillance phase we will look at the same Key Performance Indicators and metrics as we did in the past. New indicators must be developed to integrate the new elements

\section{Key Questions}

\subsection{What is the best fit in overall Industry 4.0?}

The proposed approach is being based on the manufacturing type: make to stock, make to order, assembly to order or engineering to order. A "High- Moderate- Low" scale for the assimilation of the Industry 4.0 elements, could be a good start, noting that more granularity based on specific features may focus better the decision.

\subsubsection{Make to stock: Industry 4.0 best fit}

Make to stock environment fundamentally means that manufacturer is producing goods and sells them from a finished goods inventory.

Table 1. Make to Stock Key Features

\begin{tabular}{|c|c|}
\hline Key Features & Magnitude \\
\hline Final Product variety & Low \\
\hline Demand predictability & High \\
\hline Manufacturing Changeovers & Low \\
\hline Raw materials variety & Moderate-High \\
\hline Raw materials inventory & Moderate-High \\
\hline Final product inventory levels & Moderate-High \\
\hline
\end{tabular}

This profile is characterized by low product variety, high predictability of demand and small variation, small number of changeovers during manufacturing process, moderate to high raw materials and final product inventories. Some Original Equipment Manufacturers present this kind of setup. Table 1 concentrates the main characteristics. 
Low final product variety: this characteristic automatically involves many repetitive processes / sub-processes/ operations, reason why Automation and Cyber-Physics options can be easily applied to increase productivity. Through IoT and Digitization solutions the key performance indicators can be easily tracked and improved.

High predictability of demand: this is a key requirement in a make to stock environment because of high levels of inventories engaged and related risks in case of big demand fluctuations. Cognitive Technologies and Data Analytics can be applied to learn about customer's behaviour and market needs and in this way to increase the predictability of the demand. Moderate to high inventory levels: this characteristic requires good traceability irrespective of the inventory policy applied (FIFO, LIFO, weighted average, etc.). IoT tools, Digitization and Data Analytics can be introduced to support a good traceability.

\subsubsection{Make to order: Industry 4.0 best fit}

Make to order environment is the type of manufacturing process that only starts when customer orders are received. Example of make to order environment: capital equipment manufacturers. Make to order main characteristics are summarized in Table 2.

The predictability of the demand is moderate, and Data Mining and Data Analytics may support analysing customer forecast accuracy and defining the adjustments levels.

Final product variety is moderate to high and for this reason there may be various changeovers during the production process. Repeatability may be limited because of large number of final products and this may need a cautious decision when implementing Automation and Cyber-Physics.

Through Digitization, Data Analytics and Machine Learning tools the levels of performance (inventories, line feeding, line performance, maintenance, changeovers / mix management) can be monitored live and adjusted.

Table 2. Make to order Key Features

\begin{tabular}{|c|c|}
\hline Key Features & Magnitude \\
\hline Final Product variety & Moderate-High \\
\hline Demand predictability & Moderate \\
\hline Manufacturing Changeovers & Moderate \\
\hline Raw materials variety & Low-Moderate \\
\hline Raw materials inventory & Low-Moderate \\
\hline Final product inventory levels & Low-Moderate \\
\hline
\end{tabular}

\subsubsection{Assembly to order: Industry 4.0 best fit}

Assembly to order environment has as main characteristic that the manufacturer is keeping standard components or subassemblies in inventory and is waiting the customer orders for building the final assembly. This profile presents medium predictability of demand, medium product variety, and high number of changeovers during manufacturing process, high components and sub-assembly inventories - Table 3. "Receipt of an order initiates assembly of the customized product" [4].

Example of such environment is computer manufacturing, where various components are used for producing various configurations.

In such environment, predictability of demand is low-medium as the technological evolution pace is very fast and various accessible configurations are required by customers. 
Direct link between the components in stock and the final customer's demand is a challenge in this environment.

Using Cognitive Technologies to identify the demand patterns is one option to increase the predictability of demand and avoid obsolesce.

Dealing with various product configurations, a good traceability through Digitization.

Table 3. Assembly to Order Key Features

\begin{tabular}{|c|c|}
\hline Key Features & Magnitude \\
\hline Final Product variety & Medium \\
\hline Demand predictability & Low- Medium \\
\hline Manufacturing Changeovers & High \\
\hline Raw materials variety & Moderate-High \\
\hline Raw materials inventory & Moderate-High \\
\hline Final product inventory levels & Low \\
\hline
\end{tabular}

\subsubsection{Engineering to order Industry 4.0 best fit}

This is a project-based environment where manufacturing processes are customized to deliver a unique product. Main characteristics are displayed in Table 4. Automation and Cyber-Physics may not address at its best the requirements of this environment because of its reduced number of repetitive processes/operations. Main effort being in customization and design, Data Analytics, Cognitive Technologies and Simulation Solutions may support a fast development and a solid proof of concept.

Table 4. Assembly to Order Key Features

\begin{tabular}{|c|c|}
\hline Key Features & Magnitude \\
\hline Final Product variety & High \\
\hline Demand predictability & High \\
\hline Manufacturing Changeovers & $\begin{array}{c}\text { Not applicable } \\
\text { or high }\end{array}$ \\
\hline Raw materials variety & Moderate-High \\
\hline Raw materials inventory & Moderate-High \\
\hline Final product inventory levels & Low \\
\hline
\end{tabular}

\subsubsection{Best fit in Industry 4.0: Conclusions}

Table 5 summarizes the above analysis as a starting point for a top down decision about which Industry 4.0 elements may be introduced considering the overall manufacturing environment. A detailed analysis following various particularities for each industry and organization must be performed and the best Industry 4.0 receipt must be customized. As a further step, it must be considered what common solutions are worth being implemented especially for fix cost reduction purpose- example being the smart systems to monitor energy consumption.

The Industry 4.0 elements have two roles in this exercise: they are part of the objective but they can be equally instruments to support decision making. For example, using Digital 
representation and Simulation Methods already developed and available on the market, (Digital Twins concept), various manufacturing scenarios involving Cyber Physics can be evaluated to validate the business case. Digital twins integrate IoT, AI (including Machine Learning), Data Analytics to create virtual representations of the manufacturing environment, to simulate and provide insights about their performance. Data is crucial to create these simulations and for this reason Digitization may be a pre-requisite.

Table 5. Manufacturing type and Industry 4.0 Elements Fit

\begin{tabular}{|c|c|c|c|c|c|}
\hline $\begin{array}{c}\text { Manufacturing } \\
\text { type }\end{array}$ & $\begin{array}{c}\text { Cyber- } \\
\text { Physics }\end{array}$ & Digitization & IoT & $\begin{array}{c}\text { Data } \\
\text { Analytics }\end{array}$ & $\begin{array}{c}\text { Cognitive } \\
\text { Technologies }\end{array}$ \\
\hline Make to stock & High & High & High & High & High \\
\hline Make to order & $\begin{array}{c}\text { Moderate- } \\
\text { High }\end{array}$ & Moderate & High & High & Medium-High \\
\hline Assembly to order & $\begin{array}{c}\text { Moderate- } \\
\text { Low }\end{array}$ & High & High & High & High \\
\hline $\begin{array}{c}\text { Engineering to } \\
\text { order }\end{array}$ & Low & Moderate & $\begin{array}{c}\text { Low - } \\
\text { Moderate }\end{array}$ & High & High \\
\hline
\end{tabular}

Moreover, increasing the predictability of demand through Cognitive Technologies, some of the actual manufacturing types may change (for example from Make to order to Make to stock).

\subsection{How the business case must capture the new elements to document in the best manner the course of actions, benefits and cost?}

\subsubsection{Business case definition and elements}

The business case has as ultimate purpose to convince a decision maker to agree on a certain course of actions providing relevant cost and benefit details. The business case highlights: problem statement, opportunity description, course of actions and related cost/benefit analysis.

\subsubsection{Business case for Industry 4.0 journey: main challenges}

The business case for implementing Industry 4.0 is often unbalanced by:

- high enthusiasm to enhance evolution in a fast-paced changing environment without enough arguments to generate decision;

- reluctance on the decision-making side to introduce the change and engage resources without strong benefit projection and documented risk assessment.

This is one of the reasons why some of the business cases might fail either in being rejected or in driving partially documented decision: bad decisions.

\subsubsection{Elements to be considered in the business case}

Once the best fit is identified, few factors may need special attention when the business case is developed. In most of the cases, these factors may be carrying hidden additional costs and can be grouped in a few categories (Fig. 3):

Infrastructure is usually well factored within the business case. Particularly for Industry 4.0 the following elements must be captured in the business case:

- IT infrastructure: what infrastructure is needed and what is the cost? Are the existing resources enough or need additional experts [5]? Who will drive the change (phase in 
for new equipment- phase out for old equipment): in house or externalized service? And at what costs?

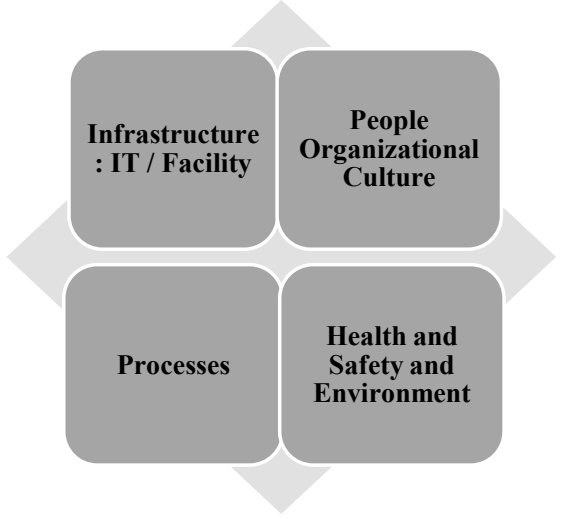

Fig. 3. Areas of focus for cost estimation

- Facility infrastructure: what layout change will be required? Are there any special requirements in terms of regulations? What is the cost of change?

People and Organizational culture: Industry 4.0 implementation requires strong cultural change that needs to be addressed through a set of actions carrying out costs. In most of the cases they refer to:

- Data management and Business Intelligence knowledge may be needed in addition to the traditional profiles.

- Training for dealing with Industry 4.0 elements: is the training available? What is the training cost? Big consultant companies such as McKinsey developed Digital Capabilities Centres where participants can experience data management and live decision making.

- How the traditional knowledge will be maintained after the implementation of the new automated tools to manage the process if manual backup is needed? With few words: how the technical community will deal with smart automation? Developing new debugging capabilities involve costs that must be considered in the business case.

- How the communication will be handled to increase awareness and eliminate the employee's concerns?

- How Industry 4.0 introduction will impact unemployment [6]?

\section{Health and Safety and Environment}

- What would be the organization's responsibility during the implementation and execution phases? Are the capabilities available or must be developed and at what costs?

- What are the regulations, how will they be implemented and what would be the cost?

Processes

- How the current processes will change? What are the pre-requisites and at what costs? How will the change be managed without disruptions?

- How the capabilities of managing new processes will be available?

- Are additional capabilities needed? How can they be achieved and how much will they cost?

- How end-to-end process knowledge will be secured?

- How the roles and responsibilities will change?

- How the overall manufacturing process will be impacted: how bottlenecks will evolve, how they can be further managed? 
- How will the Lean Six Sigma methods and tools be integrated and further developed [7]?

Building trust before Industry 4.0 capability can be easily supported by itself Industry 4.0 elements through simulation solutions as a complementary method to the traditional business case.

\subsubsection{Business case: conclusions}

Traditionally, the business case is focused on investment vs. benefit dimension. In Industry 4.0 context we need to consider that the future state cannot be achieved with the same infrastructure, organizational capabilities and mindset. The overall cost/ benefit analysis must capture all the potential implications therefore a new business case architecture may be needed. The key take away is that the new capabilities needed to deal with Industry 4.0 may be difficult to be found on the market, they must be developed with enough amount of time and budget.

\subsection{What will be the specific indicators?}

On top of traditional Indicators, Industry 4.0 implementation requires additional elements to be measured and monitored (Fig. 4):

- Data through Digitization: the availability, validity, security and relevance of the data for decision making at all levels must be indicators for a good implementation.

- Connection through IoT: The percentage of the connected elements within a system as well as the security of the connections and consequently of the data must be considered as indicators.

- The performance of predictive solutions and related actions- for example the indicators about the effects of the Predictive Maintenance.

- Decision making: the time to detect a risk or opportunity, measure, analyse and decide - must be an indicator for a successful implementation of IoT, Data Analytics and Digitization.
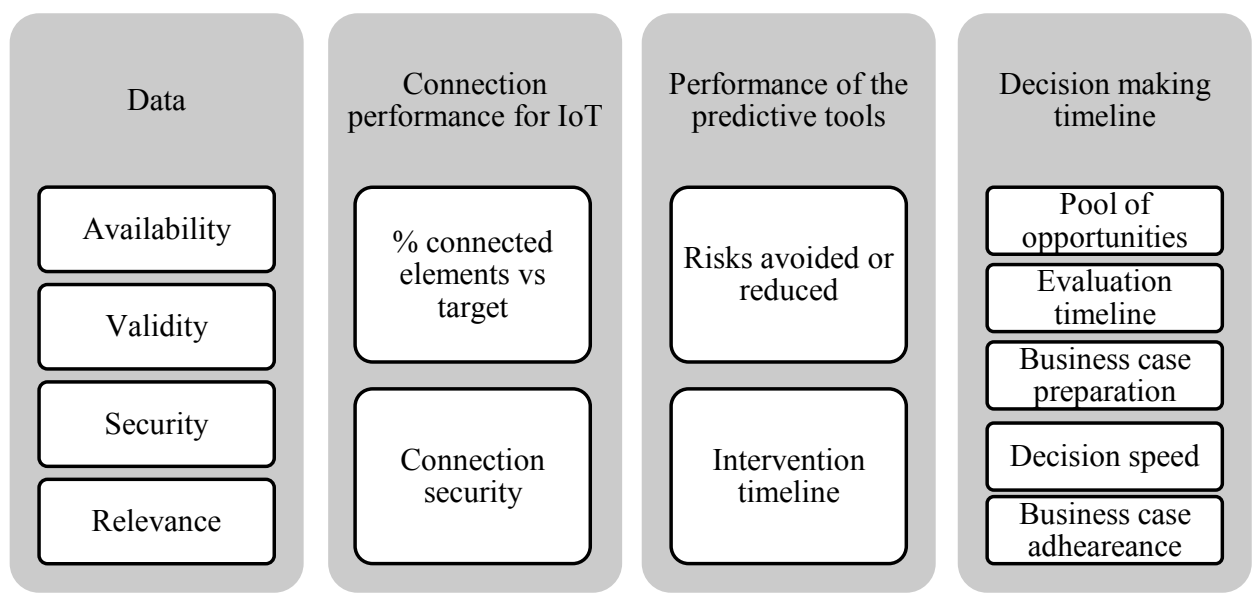

Fig. 4. Examples of elements to be measured in Industry 4.0 context

On top of measuring these indicators, another task is to understand how they influence other classic KPIs. One example specific for manufacturing is OEE [8] which is traditionally calculated based on availability, performance and quality. So far, the IoT and data technologies are already used to better measure the OEE and drive faster decisions to 
improve. The main challenge remains to re-design the OEE calculation breakdown based on the new Industry 4.0 elements.

Above indicators should be adapted and completed within Industry 4.0 environment and considered to be added to the traditional business case model. Today there are many studies available, highlighting at conceptual level the effects to be measured, however quantifiable clear indicators are still to be developed. AI/ machine learning and data mining could be used to explore the behaviour of the connected systems and the data and to suggest new indicators based on the patterns. Another aspect is how the leading vs. lagging indicators will have evolved in the future state.

\section{Conclusions}

Industry 4.0 best fit is a strategic option. Therefore, in an initial phase the top down approach proposed by this paper based on manufacturing environment would help to filter and select the future state architecture. As a next challenge and study direction it would be interesting to analyse how the solutions available in Industry 4.0. context may change the type of manufacturing environment - for example to move from Make to order into Make to stock and to reduce the overall cycle time and gain increased competitive advantage.

Business case showing the cost and benefit and proposed course of actions should evaluate carefully the implications and should consider the future state required capabilities. Developing the new capabilities must not be underestimated in terms of time and cost since their current availability is still limited. Starting with the structure of educational programs and continuing with the new jobs design and skills architecture, developing in particularly the people capabilities will be a challenging journey.

The key indicators cannot be the same as in traditional manufacturing. Despite the high range perception that Industry 4.0 is driving the improvement for actual manufacturing performance indicators, data and connectivity impact in the future state must have their own measurement methods. A future study direction will be designing the new indicators and integrating in the overall performance measurement.

\section{References}

1. O. Maksimchuk, T. Pershina, MATEC Web of Conferences, 129, 04006 (2017)

2. Deloitte, Industry 4.0 Challenges and Solutions for the Digital Transformation and Use of Digital Technologies (2014)

3. APICS, Basics of Supply Chain Management, Participant Book Version 4.1 (2013)

4. APICS, Operations Management Body of Knowledge Framework, Third Edition (A: 26.02.2019)

5. N. Norazlin, A.Y. Bani Hashim, M.H.F. M. Fauadi, Teruaki Ito, Zinvi Fu, MATEC Web of Conferences 10, 06002 (2017)

6. World Economic Forum, Employment trends (2016)

7. J. LoPresti, How Lean Six Sigma Applies to Internet of Things and Industry 4.0 (2019)

8. Alasdair Gilchrist, Industry 4.0: The Industrial Internet of Things (2016) 